\title{
Acute exacerbation of chronic obstructive pulmonary disease was associated with respiratory syncytial virus infection and the upregulation of TLR3
}

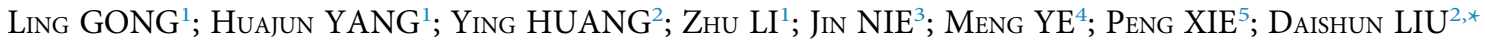 \\ ${ }^{1}$ Department of Respiratory Medicine, The First People's Hospital of Zunyi (The Third Affiliated Hospital of Zunyi Medical University), Zunyi, 563000, China \\ 2 Zunyi Medical University, Zunyi, 563000, China \\ ${ }^{3}$ Department of Respiratory Medicine, The Affiliated Hospital of Zunyi Medical University, Zunyi, 563000, China \\ ${ }^{4}$ Scientific Research Center, The First People's Hospital of Zunyi (The Third Affiliated Hospital of Zunyi Medical University), Zunyi, 563000, China \\ ${ }^{5}$ Department of Critical Care Medicine, The First People's Hospital of Zunyi (The Third Affiliated Hospital of Zunyi Medical University), Zunyi, 563000, China
}

Key words: TLR3, Chronic obstructive pulmonary disease, RSV, Inflammation

\begin{abstract}
Respiratory syncytial virus (RSV) infection is known as a risk factor for chronic obstructive pulmonary disease (COPD). RSV infection induces the upregulation of Toll-like receptor 3 (TLR3). This study aimed to investigate the association of TLR3 with RSV induced acute exacerbations of chronic obstructive pulmonary disease (AECOPD). Serum/sputum samples from AECOPD patients, stable chronic obstructive pulmonary disease (SCOPD) patients, and healthy controls were collected. Nested PCR was used to detect RSV. The lung function parameters were assessed by blood gas and lung function analysis. The expression levels of inflammatory factors in sputum and serum samples were determined by enzyme-linked immunosorbent assay. BEAS-2B cell lines were infected with RSV, and the expression of TLR3 mRNA was determined by PCR and the levels of inflammatory factors were also investigated. The presence of RSV was detected in 3 SCOPD and 8 AECOPD patients, but not in healthy patients. The expression levels of TNF- $\alpha$ and IRF- 3 in both sputum and serum samples of RSV-positive group were significantly higher than in RSV-negative group. TLR3 mRNA levels in RSV-positive group were significantly higher than those in RSVnegative group. Interestingly, the level of TLR3 mRNA expression was negatively correlated with oxygenation index and lung function parameters. Furthermore, BEAS-2B cells infected with RSV led to significant increase of the expression of TLR3 mRNA and inflammatory factors IFN- $\beta$, IL-13, IL-32, and TNF- $\alpha$. Our observations indicate that AECOPD is associated with RSV infection and the upregulation of TLR3.
\end{abstract}

\section{Introduction}

Chronic obstructive pulmonary disease (COPD) is a common chronic disease with high mortality and morbidity (Ford et al., 2015). The incidence of COPD in Chinese over 40 years old was as high as 13.7\% (Wang et al., 2018). Although the diagnosis and treatment of COPD have significantly improved, the survival of patients remains poor due to COPD-induced irreversible lung dysfunction (Lange et al., 2015). Airway remodeling is a typical pathological feature of COPD, and neutrophils are the main effector cells. Lymphocytes are the primary cells implicated in airway inflammation, and the percentages of $\mathrm{CD}^{+}$cells

*Address correspondence to: Daishun Liu, ldslwtg@126.com Received: 09 July 2021; Accepted: 30 August 2021 significantly increase (Polosukhin et al., 2017). Protease causes the destruction of elastin, the main component of connective tissues of lung parenchyma, leading to tissue damage and airway remodeling in COPD (Chotirmall et al., 2015). Harmful substances stimulate the vagus nerve and cause the release of acetylcholine, leading to excessive secretion of airway mucus, smooth muscle contraction, and airway spasm (Mirza et al., 2018; Morélot-Panzini et al., 2016). Various predisposing factors have been identified for COPD, such as respiratory syncytial virus (RSV) (Clark et al., 2014; Coultas et al., 2019). RSV is an independent risk factor for COPD patients in Intensive Care Unit (ICU) (Friedman et al., 2017; Marçôa et al., 2018; Mehta et al., 2013).

Toll-like receptors (TLRs) are key components of innate and adaptive immune system, and have been implicated in tumor progression, inflammation, and autoimmune diseases (Alexander et al., 2016). TLR3 and TLR4 recruit the adaptor 
protein TRIF through MyD88 independent pathway to activate interferon regulatory factor 3 (IRF-3) and induce the production of interferon-I (IFN-I), causing the secretion of interferon- $\beta$ (INF- $\beta$ ) (Alexander et al., 2016). As a result, the expression of IRF3, a TLR3 downstream signaling factor, increases and it activates nuclear factor $\kappa \mathrm{B}(\mathrm{NF}-\kappa \mathrm{B})$ (Brownell et al., 2014; Chen et al., 2015). NF- $\kappa \mathrm{B}$ activation causes the upregulation of inflammatory interleukins and tumor necrosis factor- $\alpha$ (TNF- $\alpha$ ) (Tsubaki et al., 2015). The excessive release of inflammatory factors can cause secondary immune injury (Hong et al., 2020). Therefore, this study aimed to investigate the association of TLR3 with RSV induced acute exacerbations of chronic obstructive pulmonary disease (AECOPD).

\section{Materials and Methods}

\section{Patients}

This study was approved by the Ethics Committee of the Third Affiliated Hospital of Zunyi Medical University (Approval No. 20354). A total of 90 participants were enrolled, including 30 healthy controls and 60 COPD patients. The patients were hospitalized in the Department of Respiratory Medicine of the First People's Hospital of Zunyi City from October 2016 to March 2017. The inclusion and exclusion criteria were given below:

\section{Inclusion criteria}

a) According to GOLD COPD classification and grouping guidelines (Lee et al., 2013), we selected those participants who, after inhaling short-acting bronchodilators, had forced expiratory volume in one second $\left(\mathrm{FEV}_{1}\right)$ to forced vital capacity (FVC) ratio of less than $70 \%$, percentage of predicted $\mathrm{FEV}_{1}$ of less than 80 , and the reversibility of airflow limitation of less than $15 \%$ of the baseline value and/or $<200 \mathrm{~mL}$.

b) AECOPD group (30 cases): had any two symptoms out of exacerbated dyspnea, increased sputum and increased sputum volume, and the symptoms persisted for more than 2 days, or had any one of the above symptoms accompanied with one of these symptoms: wheezing, cough, sore throat and common cold.

c) Stable chronic obstructive pulmonary disease (SCOPD) group (30 cases): were asymptomatic and not in the acute phase for more than 30 days and did not take any treatment (inhalation or oral medication) for the 14 days before enrollment.

d) Healthy group (30 cases): had no COPD, pneumonia, bronchial asthma, bronchiectasis, pulmonary tuberculosis, or any other respiratory disease, nor had any serious disease of the heart, liver, kidney, and blood, nor any systemic disease.

e) Exclusion criteria: patients with respiratory diseases other than COPD such as bronchial asthma, bronchiectasis, and pneumonia. Patients with tuberculosis or any serious heart, liver, kidney, blood, and systemic disease were also excluded.

\section{Data collection}

The age, gender, lung function parameters $\left(\mathrm{FEV}_{1}, \mathrm{FVC}\right.$, $\mathrm{FEV}_{1} / \mathrm{FVC}$ ), arterial blood gas analysis $\mathrm{PH}$ value (hydrogen ion concentration), oxygenation index, carbon dioxide partial pressure, white blood cells, and neutrophils ratio of subjects were recorded. Sputum samples were collected within $24 \mathrm{~h}$ of admission; sputum from participants showing little or no sputum was collected by induced sputum excretion (Tables 1 and 2).

\section{Cell culture and infection}

Human bronchial epithelial cell line (BEAS-2B) (Cat \# CRL9609) and RSV standard strain (Long) (Cat \#ATCC VR-26) were purchased from American Type Culture Collection, RSV Long strain was first isolated by Robert Chanock from a child with bronchopneumonia in 1956 and was subsequently passaged 11 to 13 times in HEp-2 cells. It is the "first" prototypic strain of RSV (Pandya et al., 2019). BEAS-2B cells were cultured in DMEM/F12 medium (Hyclone, USA) supplemented with $100 \mathrm{U} / \mathrm{mL}$ penicillin and $25 \mathrm{mg} / \mathrm{mL}$ gentamicin and incubated at $37^{\circ} \mathrm{C}$ with $5 \%$ $\mathrm{CO}_{2}$. BEAS-2B cells were infected with RSV at multiplicity of infection (MOI) of 1, 0.3, 0.1, and 0.01 (Liu et al., 2018). Prime Script RT reagent kits (Cat \# RR0370) and One-Step TB Green PrimeScript RT-PCR Kit (Cat \# RR086B) were obtained from TaKaRa (Dalian, China). PCR primers were provided by TaKaRa (the sequences were listed in Table 3 ).

\section{ELISA}

The levels of IFN- $\beta$, IRF3, TNF- $\alpha$, interleukin (IL)-13, IL-32 in serum or cell supernatants were detected by ELISA kits (R\&D Systems, USA) following the manufacturer's protocols.

\section{Statistical analysis}

The data were expressed as mean \pm standard deviation (SD). $T$-test was used for the data between the two groups with normal distribution, and rank sum test was used for the data between the two groups without normal distribution. Variance analysis was used for comparison among multiple groups. Pearson correlation analysis was used, and Pearson correlation was expressed by $r$. Categorical variables were reported as frequency and percentage. Data were processed and analyzed using Microsoft Excel 2010 and SPSS statistical software (version 19.0, SPSS Inc., Chicago, Illinois), and $P<$ 0.05 was considered as statistically significant.

\section{Results}

Detection of RSV in clinical samples

Since RSV is an independent risk factor for COPD patients (Friedman et al., 2017; Marçôa et al., 2018; Mehta et al., 2013), we detected RSV. The participants included in our study were categorized into 3 groups $(\mathrm{N}=30)$ : healthy control group, AECOPD group, and SCOPD group. RSV was not detected in healthy group, while RSV was detected in 3 SCOPD patients (3/30) and 8 AECOPD patients (8/30).

The levels of TLR3 and inflammatory factors in clinical samples The expression of TLR3 mRNA in the sputum of COPD patients increased, and the levels in the SCOPD group were higher than those in healthy group. TLR3 mRNA levels in the AECOPD group were significantly higher than the corresponding levels in the SCOPD group. Furthermore, RSV positive group showed significantly higher TLR3 mRNA levels than RSV negative group (Fig. 1A). TLR3 
TABLE 1

Clinical data of RSV-positive and RSV-negative COPD patients

\begin{tabular}{|c|c|c|c|}
\hline Basic information & $\begin{array}{l}\text { RSV-positive }(\mathrm{N}=11) \\
(\mathrm{AECOPD}=8, \mathrm{SCOPD}=3)\end{array}$ & $\begin{array}{l}\text { RSV-negative }(N=49) \\
(A E C O P D=22, \text { SCOPD = 27) }\end{array}$ & $P$-value \\
\hline Age & $70.2 \pm 8.21$ & $68.9 \pm 7.87$ & 0.63 \\
\hline \multicolumn{4}{|l|}{ Lung function } \\
\hline FEV1, L & $0.96 \pm 0.32$ & $1.12 \pm 0.48$ & 0.297 \\
\hline FVC, L & $1.42 \pm 0.71$ & $1.87 \pm 0.68$ & 0.054 \\
\hline FEV1\% predicted & $47.34 \pm 8.32$ & $57.54 \pm 14.8$ & $0.031^{\star}$ \\
\hline FEV1/FVC\% & $57.23 \pm 5.64$ & $61.76 \pm 5.43$ & $0.015^{\star}$ \\
\hline \multicolumn{4}{|l|}{ Blood } \\
\hline $\mathrm{PO}_{2}, \mathrm{mmHg}$ & $59.59 \pm 3.35$ & $65.87 \pm 10.43$ & 0.054 \\
\hline $\mathrm{PCO}_{2}, \mathrm{mmHg}$ & $41.3 \pm 9.54$ & $38.7 \pm 5.43$ & 0.223 \\
\hline WBC, $10^{9} / \mathrm{L}$ & $8.34 \pm 0.54$ & $8.54 \pm 1.54$ & 0.674 \\
\hline N\% & $82.54 \pm 3.56$ & $80.32 \pm 2.34$ & $0.012^{\star}$ \\
\hline IL-6, pg/mL & $25.63 \pm 19.42$ & $15.42 \pm 10.31$ & $0.016^{*}$ \\
\hline PCT, ng/mL & $0.11 \pm 0.05$ & $0.1 \pm 0.04$ & 0.477 \\
\hline
\end{tabular}

Note: Data expressed as mean \pm SD. FEV1 means forced expiratory volume in one second. L means liter. FVC means forced vital capacity. WBC means white blood count. IL-6 means interleukin-6. PCT means procalcitonin. ${ }^{\star} P<0.05$ indicated significant difference.

\section{TABLE 2}

Basic clinical data of the subjects

\begin{tabular}{|c|c|c|c|}
\hline Basic information & Healthy control $(\mathrm{N}=30)$ & $\begin{array}{l}\text { SCOPD } \\
(\mathbf{N}=30)\end{array}$ & $\begin{array}{l}\text { AECOPD } \\
(\mathbf{N}=30)\end{array}$ \\
\hline Age (range) & $60.4(39-75)$ & $72.17(51-86)$ & $70.3(39-88)$ \\
\hline Male/Female, N (\%) & $18(60 \%) / 12(40 \%)$ & $23(77 \%) / 7(23 \%)$ & $22(73 \%) / 8(27 \%)$ \\
\hline \multicolumn{4}{|l|}{ Smoking habit } \\
\hline Never smoke, N (\%) & $12(40 \%)$ & $4(13 \%)$ & $2(7 \%)$ \\
\hline Quit smoking, N (\%) & $9(30 \%)$ & $5(17 \%)$ & $10(33 \%)$ \\
\hline Current, N (\%) & $9(30 \%)$ & $21(70 \%)$ & $18(60 \%)$ \\
\hline \multicolumn{4}{|l|}{ GOLD severity class, N (\%) } \\
\hline 1 & & $2(7 \%)$ & $4(13 \%)$ \\
\hline 2 & & $13(43 \%)$ & $11(37 \%)$ \\
\hline 3 & & $9(30 \%)$ & $9(30 \%)$ \\
\hline 4 & & $6(20 \%)$ & $6(20 \%)$ \\
\hline \multicolumn{4}{|l|}{ Lung function } \\
\hline FEV1, L (range) & & $1.14(1.45-2.27)$ & $1.08(0.54-1.58)$ \\
\hline FVC, L (range) & & $1.85(1.6-3.48)$ & $1.35(0.8-1.77)$ \\
\hline FEV1\%predicted (range) & & $63.06(50.76-68.36)$ & $44.06(26.5-58.98)$ \\
\hline FEV1/FVC\% (range) & & $62.69(51.23-69.2)$ & $51.99(42.05-59.88)$ \\
\hline \multicolumn{4}{|l|}{ Blood } \\
\hline $\mathrm{PO}_{2}, \mathrm{mmHg}$ (range) & & $73.22(32.6-91.1)$ & $72.60(38.4-90.7)$ \\
\hline $\mathrm{PCO}_{2}, \mathrm{mmHg}$ (range) & & $34.25(23.3-64.6)$ & $39.85(18.9-74.1)$ \\
\hline WBC, $10^{9} / \mathrm{L}$ (range) & & $8.08(2.8-14.8)$ & $8.3(3.3-18.2)$ \\
\hline N\% (range) & & $74.27(43.5-92.4)$ & $76.65(57.9-94.8)$ \\
\hline IL-6, pg/ml (range) & & $6.11(2.2-48.1)$ & $10.29(1.5-50.7)$ \\
\hline PCT, ng/ml (range) & & $0.067(0.02-0.173)$ & $0.097(0.42-0.373)$ \\
\hline
\end{tabular}

Note: N\% means percent of neutrophile granulocyte. 
TABLE 3

Primer sequences

\begin{tabular}{lll}
\hline Primer sequence of genes $\left(\mathbf{5}^{\prime}-\mathbf{3}^{\prime}\right.$ ) & Amplification length (bp) \\
\hline $\begin{array}{l}\text {-actin (forward) } \\
\beta \text {-actin (reverse) }\end{array}$ & AGCGAGCATCCCCCAAAGTT & 285 \\
TLR3 (forward) & GCAACAACAACATAGCCAACAT & \multirow{2}{*}{153} \\
TLR3 (reverse) & GGAGGTGAGACAGACCCTTTAG & \\
RSV G (forward) & The first walking CCATTCTGGCAATGATAATCTC & 450 \\
RSV G (reverse) & The first walking GTTTTTTGTTTGGTATTCTTTTGCAG & \\
RSV G (forward) & The second walking CGGCAAACCACAAAGTCACAC & 326 \\
RSV G (reverse) & The second walking GGGTACAAAGTTAAACACTTC & \\
\hline
\end{tabular}

(A)

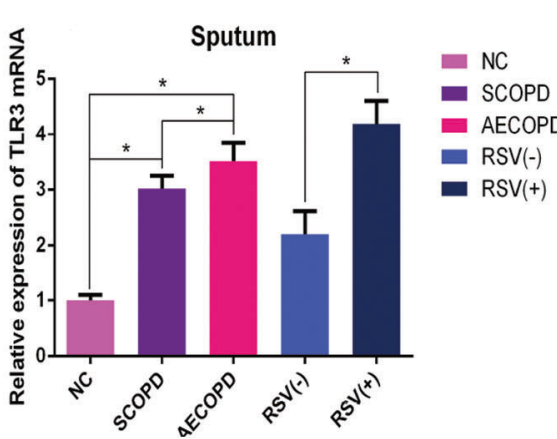

(C)

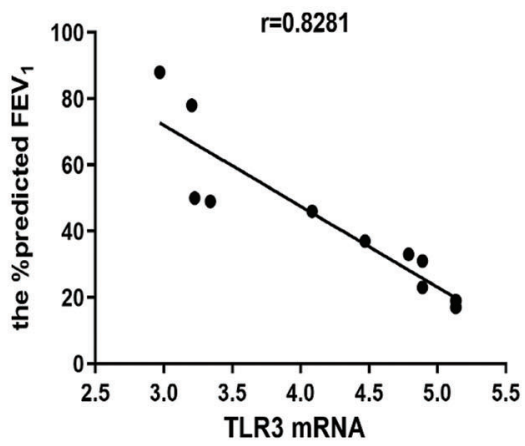

(B)

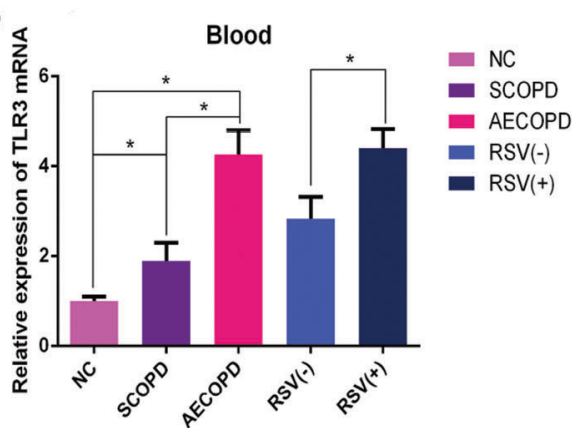

(D)

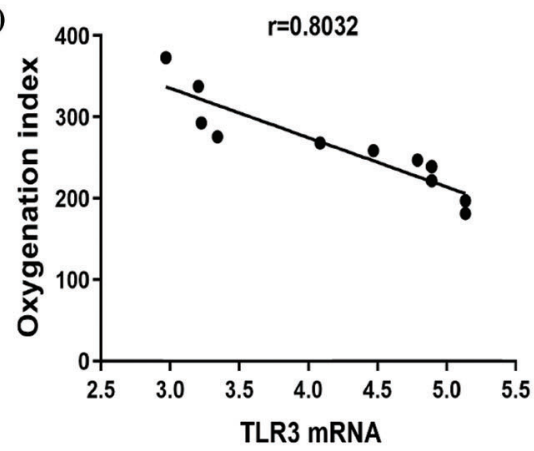

FIGURE 1. The relationship between TLR3 mRNA expression and lung function parameters. (A) TLR3 mRNA expression levels in sputum from SCOPD group ( $\mathrm{N}=30$ ), AECOPD group $(\mathrm{N}=30)$, RSV-positive group $(\mathrm{N}=11)$, RSV-negative group $(\mathrm{N}=49)$ and $\mathrm{NC}$ (healthy control) group $(\mathrm{N}=30)$, respectively. (B) TLR3 mRNA expression levels in serum samples of SCOPD group $(\mathrm{N}=30)$, AECOPD group $(\mathrm{N}=30)$, RSV-positive group $(\mathrm{N}=11)$, RSV negative group $(\mathrm{N}=49)$, and $\mathrm{NC}$ group $(\mathrm{N}=30)$, respectively. $(\mathrm{C}-\mathrm{D})$ Correlation of TLR3 mRNA expression and FEV1 and the oxygenation index in RSV-positive patients. ${ }^{*} P<0.05$.

mRNA expression levels in blood were similar to those in the sputum (Fig. 1B). Pearson correlation analysis showed that increased expression of TLR3 mRNA in the sputum of RSVpositive patients was negatively correlated with FEV1 (Fig. 1C, $\mathrm{r}=-0.8281$ ) and oxygenation index (Fig. 1D, $\mathrm{r}=$ $-0.8032)\left(\right.$ All $\left.{ }^{\star} P<0.05\right)$.

In addition, the levels of inflammatory factors (IFN- $\beta$, IRF3, TNF- $\alpha$, IL-13, and IL-32) were higher in the sputum of COPD and RSV-positive patients than in healthy group (Fig. 2) $\left(\right.$ All $\left.{ }^{\star} P<0.05\right)$. Similarly, the levels of inflammatory factors were higher in the blood of COPD and RSV-positive patients than in healthy group (Fig. 3 ; all ${ }^{\star} P<0.05$ ).

Changes in TLR3 $m R N A$ and inflammatory factors in $B E A S-2 B$ cells infected with RSV

Next, we selected optimal concentration and time for the infection of BEAS-2B cells by RSV without causing excessive cell death. The viability of BEAS-2B cells after RSV infection was shown in Fig. 4A. With time of RSV infection as $12 \mathrm{~h}$ and $24 \mathrm{~h}$, the increase in the MOI of virus (MOI $=0.01$, MOI $=0.3$ ) significantly increased the expression of inflammatory factors such as IFN- $\beta$ (Fig. 4B), TNF- $\alpha$ (Fig. 4C), IL-13 (Fig. 4D), and IL-32 (Fig. 4E). In addition, the longer the infection by RSV, the higher expression of TLR3 mRNA (Figs. 4F and 4G).

\section{Discussion}

In present study, sputum samples from 90 patients were tested for RSV by nested PCR. RSV was not detected in 30 control subjects; RSV was detected in 11 of 60 COPD patients (18.3\%). This percentage was lower compared to previous study (Anderson et al., 2016), and the variation may be due to the difference in regional characteristics. Among the 30 

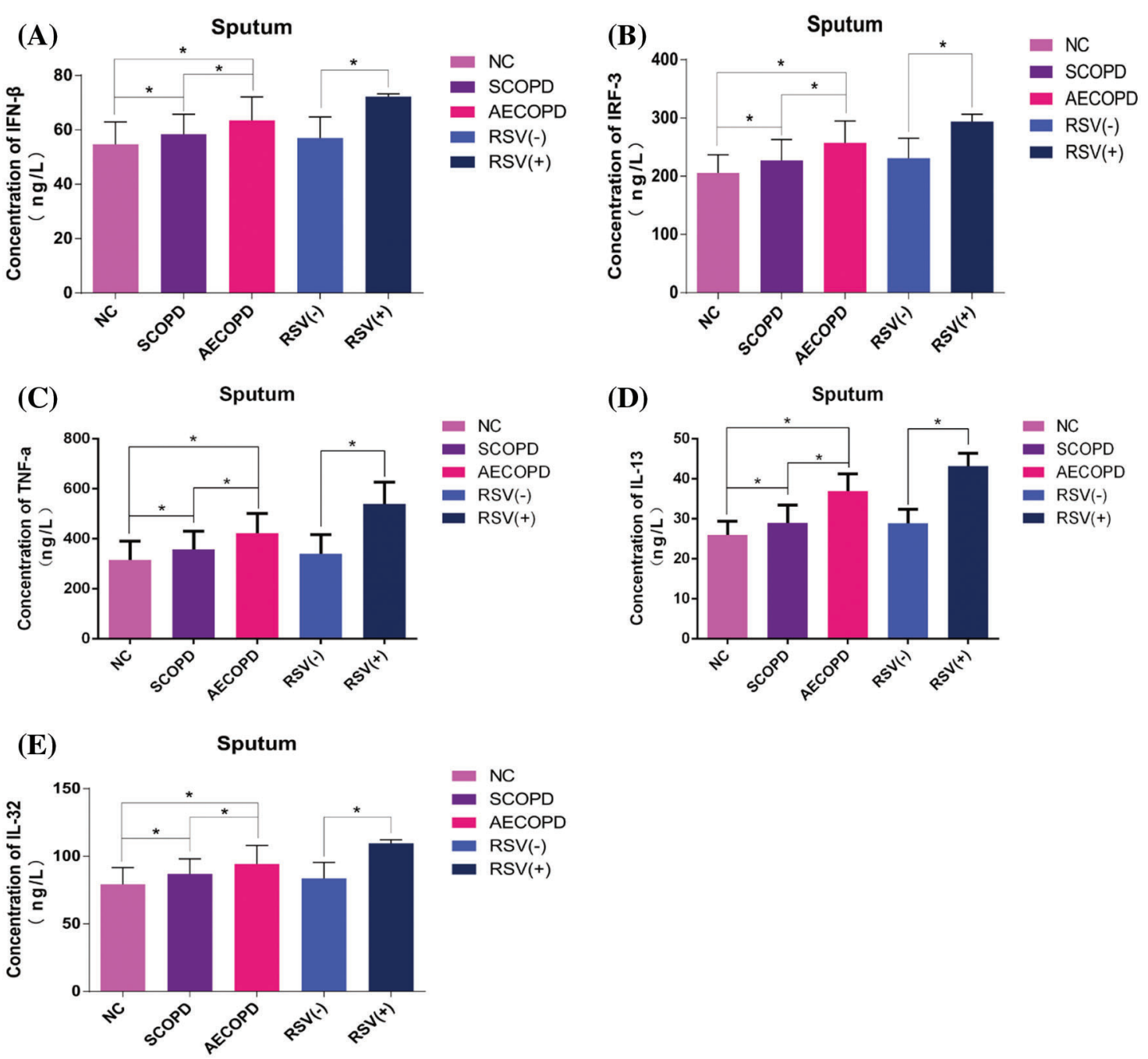

FIGURE 2. Expression levels of IFN- $\beta$, IRF3, TNF- $\alpha$, IL-13, and IL-32 in sputum determined by ELISA. (A) Expression levels of IFN- $\beta$, (B) Expression levels of IRF-3, (C) Expression levels of TNF- $\alpha$, (D) Expression levels of IL-13, (E) Expression levels of IL-32 in SCOPD group (N = $30)$, NC (healthy control) group $(\mathrm{N}=30)$, AECOPD group $(\mathrm{N}=30)$, RSV positive group $(\mathrm{N}=11)$, and RSV negative group $(\mathrm{N}=49) .{ }^{\star} P<0.05$.

SCOPD patients, only three were RSV-positive (10\%), while eight out of 30 AECOPD patients were RSV-positive (26.6\%). The main cause of AECOPD attacks may be the presence of latent and colonized RSV in the airway of COPD patients.

TLR3 plays a regulatory role by recognizing doublestranded RNA (dsRNA) produced after viral infection (Wang et al., 2016). The dsRNA is a replication intermediate of most RNA viruses such as RSV, and it is able to induce the upregulation of TLR3 expression (Liu et al., 2018). In this study we performed PCR and found that TLR3 mRNA levels in the sputum of COPD patients were significantly higher than the levels in the sputum of the normal group, while TLR3 mRNA levels of AECOPD group patients were significantly higher than those of SCOPD group. Meanwhile, the expression levels of TLR3 mRNA in RSV-positive group were significantly higher than the levels in RSV-negative group. The expression levels of TLR3 mRNA in blood showed a similar trend. The expression of TLR3 mRNA in the sputum samples of RSV positive patients showed a negative correlation with FEV1 ( $\mathrm{r}=$ $0.8281)$ and oxygenation index $(r=0.8032)$. A recent study based on TLR3-deficient mice reported that viral infection led to the upregulation of TLR3 in monocyte-derived dendritic cells, which would drive type 2 immune response to cause chronic inflammatory diseases of the lung such as COPD (Wang et al., 2021). These results suggest that TLR3 is required to mediate RSV induced COPD and its expression level is related to the severity of COPD.

Next, we used ELISA to detect the expression levels of IFN- $\beta$, IRF3, TNF- $\alpha$, IL-13, and IL-32 in the blood, and found that COPD group had higher expression levels of IFN- $\beta$, IRF3, TNF- $\alpha$, IL-13, and IL-32 than the control group. The expression levels of IRF3, TNF- $\alpha$, IL-13, and IL32 in RSV-positive group were significantly higher than the levels in RSV-negative group $(P<0.05)$. Previous studies have shown that the expression levels of inflammatory factors CCL3 and CCL5 in the bronchoalveolar lavage fluid were significantly increased in RSV-infected mouse models (Salimi et al., 2017). Our study found that with the increase in RSV infection time and virus titer, the expression of inflammatory factors IFN- $\beta$, TNF- $\alpha$, IL-13, and IL-32 significantly increased. These observations suggest that in COPD patients, especially those with RSV infection, 


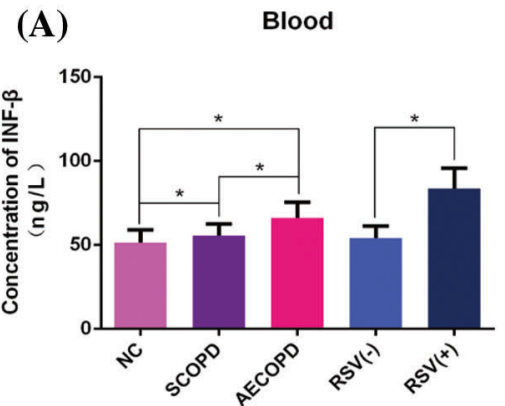

(C)

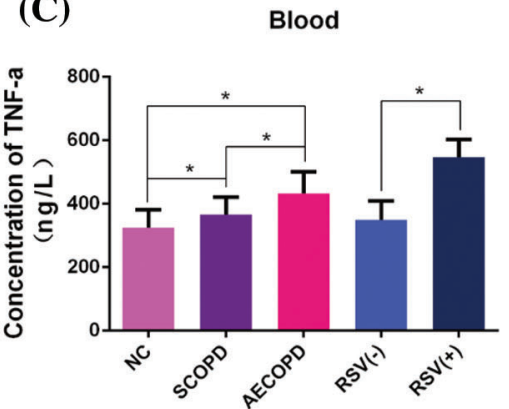

(E)

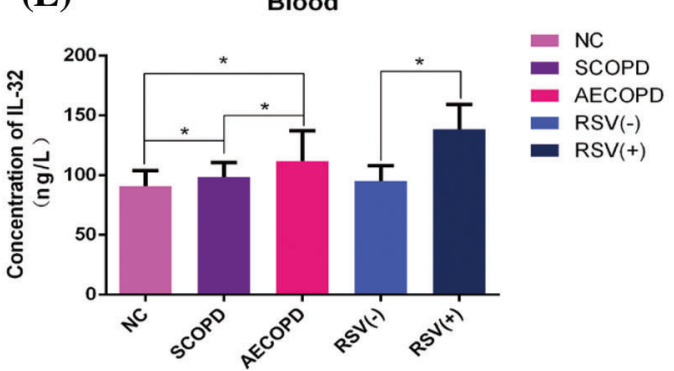

(B)

Blood

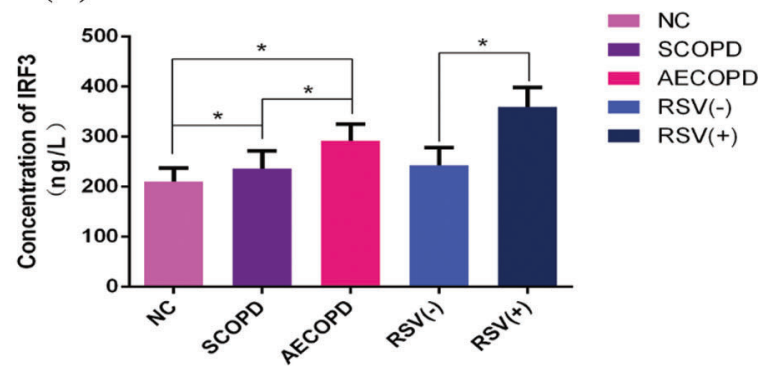

(D)

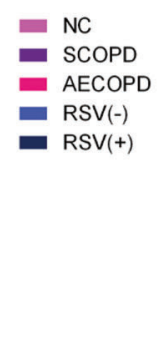

FIGURE 3. Expression levels of IFN- $\beta$, IRF3, TNF- $\alpha$, IL-13, and IL-32 in serum determined by ELISA. (A) Expression levels of IFN- $\beta$, (B) Expression levels of IRF-3, (C) Expression levels of TNF- $\alpha$, (D) Expression levels of IL-13, (E) Expression levels of IL-32 in SCOPD group (N = $30), \mathrm{NC}$ (healthy control) group $(\mathrm{N}=30)$, AECOPD group $(\mathrm{N}=30)$, RSV positive group $(\mathrm{N}=11)$ and RSV negative group $(\mathrm{N}=49)$. ${ }^{\star} P<0.05$.

increased levels of inflammatory factors cause a series of inflammatory reactions and inducing immune injury (Foronjy et al., 2014; Na et al., 2020). In addition, TLR3 mediates the production of various inflammatory factors, inducing a series of inflammatory responses and aggravating the immune injury to the lungs. A recent study showed that TLR3 inhibitor could reduce mRNA and protein expression of various inflammatory cytokines and attenuate cigarette smoke induced airway inflammation (Jiang et al., 2021).
Therefore, we speculate that RSV induced upregulation of TLR3 may contribute to airway inflammation and remodeling in COPD.

In summary, our results suggest that inflammatory response induced by RSV is related to the upregulation of TLR3, and it may cause secondary lung immune injury in AECOPD patients. The levels of inflammatory factors in serum and sputum can indicate the severity of COPD and are helpful for treatment evaluation and prognosis judgment. 
(A)

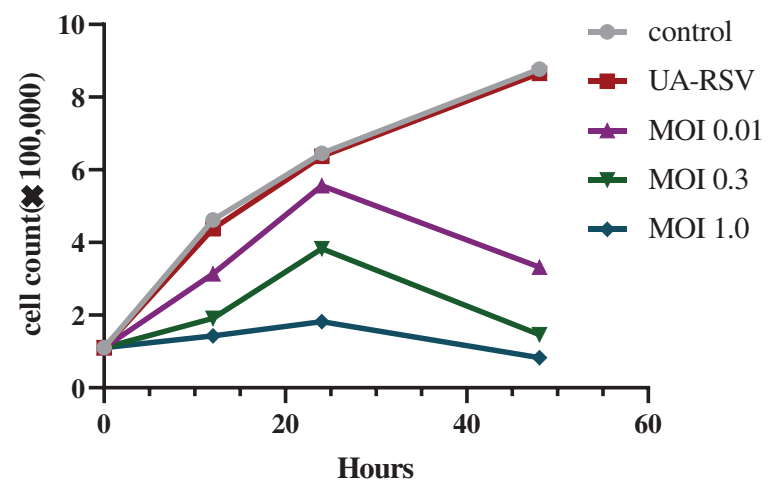

(B)

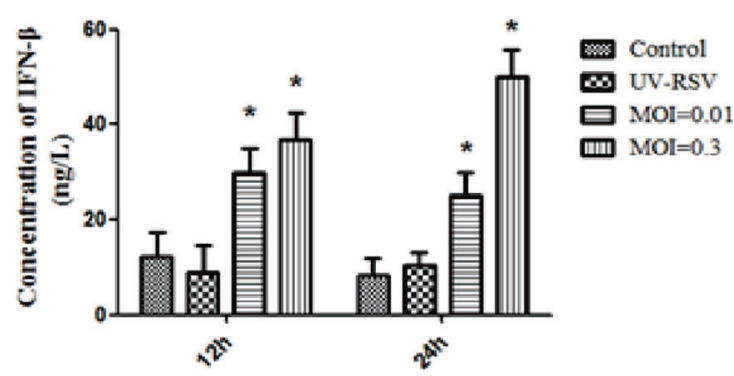

(D)

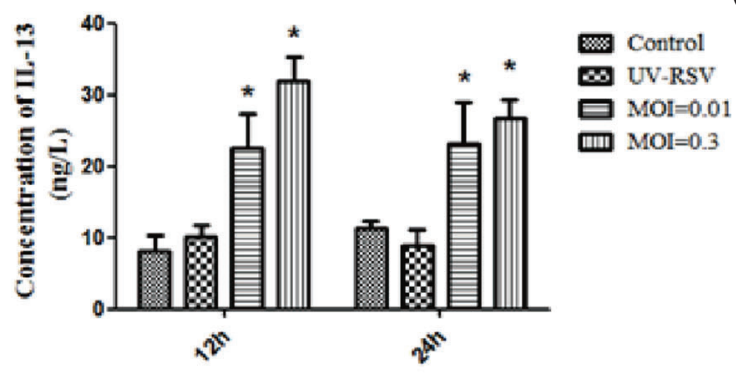

(F)

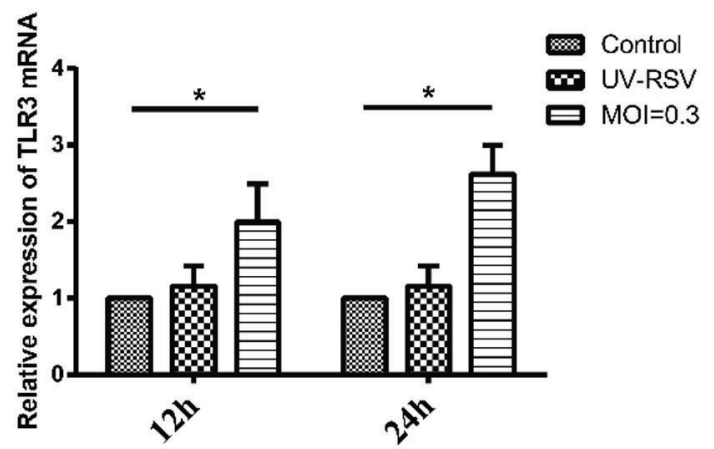

(C)

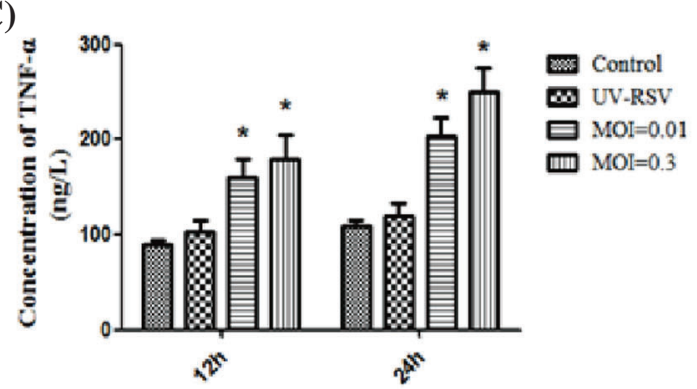

(E)

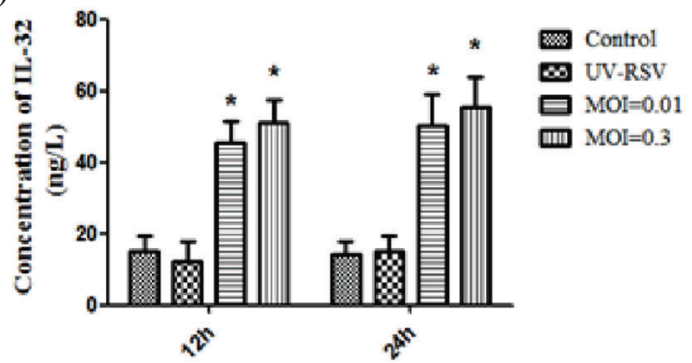

(G)

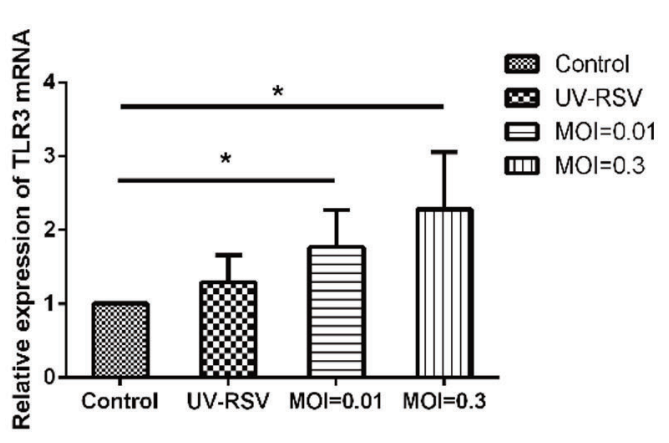

FIGURE 4. The viability and the expression levels of inflammatory factors and TLR3 of BEAS-2B cells after infection with RSV at different time $(12 \mathrm{~h}, 24 \mathrm{~h}$ ) and MOI. (A) The viability of BEAS-2B cells after RSV infection. (B-E) The expression levels of inflammatory factor IFN- $\beta$, TNF- $\alpha$, IL-13, and IL-32 at different time points $(12 \mathrm{~h}, 24 \mathrm{~h}$ ), when BEAS-2B cells were infected with viruses at MOI $=0.01,0.3$. (F-G) Changes in TLR3 mRNA expression levels at different time point $(12 \mathrm{~h}, 24 \mathrm{~h})$, when BEAS-2B cells were infected with viruses at $\mathrm{MOI}=0.01,0.3 .{ }^{\star} P<0.05$.

Availability of Data and Materials: The datasets generated during and/or analyzed during the current study are available from the corresponding author on reasonable request.

Author Contribution: Study conception and design: Daishun Liu, Ling Gong; data collection: Huajun Yang, Ying Huang, $\mathrm{Zhu} \mathrm{Li}$; analysis and interpretation of results: Jin Nie, Meng Ye, Peng Xie; draft manuscript preparation: Ling Gong. All authors reviewed the results and approved the final version of the manuscript.

Ethics Approval: This study was approved by the Ethics Committee of the Third Affiliated Hospital of Zunyi Medical University (Approval No. 20354, July 2016).

Funding Statement: This work was supported by Natural Science Foundation of China (No. 82060010), Guizhou Provincial Natural Science Foundation Project ([2018] 5623), Guizhou Provincial Respiratory Critical Disease Clinical Research and Prevention and Treatment Talent Base Project ([2020]8), Zunyi Respiratory Medicine Talent Base Project ([2019]69), Science and Technology Bureau Project of Zunyi City (Zunshi Kehe HZ[2020]3 and [2020]292). 
Conflicts of Interest: The authors declare that they have no conflicts of interest to report regarding the present study.

\section{References}

Alexander CM, Xiong KN, Velmurugan K, Xiong J, Osgood RS et al. (2016). Differential innate immune cell signatures and effects regulated by toll-like receptor 4 during murine lung tumor promotion. Experimental Lung Research 42: 154-173. DOI 10.3109/01902148.2016.1164263.

Anderson NW, Binnicker MJ, Harris DM, Chirila RM, Brumble L et al. (2016). Morbidity and mortality among patients with respiratory syncytial virus infection: A 2-year retrospective review. Diagnostic Microbiology and Infectious Disease 85: 367-371. DOI 10.1016/j.diagmicrobio.2016.02.025.

Brownell J, Bruckner J, Wagoner J, Thomas E, Loo YM et al. (2014). Direct, interferon-independent activation of the CXCL10 promoter by $\mathrm{NF}-\kappa \mathrm{B}$ and interferon regulatory factor 3 during hepatitis $C$ virus infection. Journal of Virology 88: 1582-1590. DOI 10.1128/JVI.02007-13.

Clark TW, Medina MJ, Batham S, Curran MD, Parmar S et al. (2014). Adults hospitalised with acute respiratory illness rarely have detectable bacteria in the absence of COPD or pneumonia; viral infection predominates in a large prospective UK sample. Journal of Infection 69: 507-515. DOI 10.1016/j.jinf.2014.07.023.

Coultas JA, Smyth R, Openshaw PJ (2019). Respiratory syncytial virus (RSV): A scourge from infancy to old age. Thorax 74 : 986-993. DOI 10.1136/thoraxjnl-2018-212212.

Chen ZG, Luo H, Wang SC, Xu JY, Li JX (2015). Antiviral effects of Jinxin oral liquid against respiratory syncytial virus infection in the BALB/C mice model. Journal of Ethnopharmacology 162: 287-295. DOI 10.1016/j.jep.2015.01.002.

Chotirmall SH, Al-Alawi M, Mcenery T, Mcelvaney NG (2015). Alpha-1 proteinase inhibitors for the treatment of alpha-1 antitrypsin deficiency: Safety, tolerability, and patient outcomes. Therapeutics and Clinical Risk Management 11: 143. DOI $10.2147 /$ TCRM.

Ford ES, Murphy LB, Khavjou O, Giles WH, Holt JB et al. (2015). Total and state-specific medical and absenteeism costs of COPD among adults aged 18 years in the United States for 2010 and projections through 2020. Chest 147: 31-45. DOI 10.1378/chest.14-0972.

Foronjy RF, Dabo AJ, Taggart CC, Weldon S, Geraghty P (2014). Respiratory syncytial virus infections enhance cigarette smoke induced COPD in mice. PLoS One 9: e90567. DOI 10.1371/journal.pone.0090567.

Friedman D, Fryzek J, Jiang X, Bloomfield A, Ambrose CS et al. (2017). Respiratory syncytial virus hospitalization risk in the second year of life by specific congenital heart disease diagnoses. PLoS One 12: e0172512. DOI 10.1371/journal.pone.0172512.

Hong Y, Wan B, Li X (2020). Age-related modifications of macrophages influenced by inflammageing in graft $v s$. host disease. BIOCELL 44: 237.

Jiang JJ, Chen SM, Li HY, Xie QM, Yang YM (2021). TLR3 inhibitor and tyrosine kinase inhibitor attenuate cigarette smoke/poly I: C-induced airway inflammation and remodeling by the EGFR/TLR3/MAPK signaling pathway. European Journal of Pharmacology 890: 173654.

Lange P, Celli B, Agustí A, Boje Jensen G, Divo M et al. (2015). Lung-function trajectories leading to chronic obstructive pulmonary disease. New England Journal of Medicine 373: $111-122$.
Lee N, Lui G, Wong K, Li T, Tse E et al. (2013). High morbidity and mortality in adults hospitalized for respiratory syncytial virus infections. Clinical Infectious Diseases 57: 1069-1077.

Liu D, Chen Q, Zhu H, Gong L, Huang Y et al. (2018). Association of respiratory syncytial virus toll-like receptor 3-mediated immune response with COPD exacerbation frequency. Inflammation 41: 654-666.

Marçôa R, Rodrigues DM, Dias M, Ladeira I, Vaz AP et al. (2018). Classification of chronic obstructive pulmonary disease (COPD) according to the new Global Initiative for Chronic Obstructive Lung Disease (GOLD) 2017: Comparison with GOLD 2011. Journal of Chronic Obstructive Pulmonary Disease 15: 21-26. DOI 10.1080/15412555.2017.1394285.

Mehta J, Walsh EE, Mahadevia PJ, Falsey AR (2013). Risk factors for respiratory syncytial virus illness among patients with chronic obstructive pulmonary disease. Journal of Chronic Obstructive Pulmonary Disease 10: 293-299. DOI 10.3109/ 15412555.2012.744741.

Mirza S, Clay RD, Koslow MA, Scanlon PD (2018). COPD guidelines: A review of the 2018 GOLD report. Mayo Clinic Proceedings 93: 1488-1502. DOI 10.1016/j.mayocp.2018.05.026.

Morélot-Panzini C, Gilet H, Aguilaniu B, Devillier P, Didier A et al. (2016). Real-life assessment of the multidimensional nature of dyspnoea in COPD outpatients. European Respiratory Journal 47: 1668-1679. DOI 10.1183/13993003.01998-2015.

Na E, Choi M, Park I, Lim S (2020). Effect of Black Sea bream extracts on cytokine production in lipopolysaccharide-induced inflammation. Biocell 44: 193-199. DOI 10.32604/ biocell.2020.08648.

DuPolosukhin VV, Richmond BW, Du RH, Cates JM, Wu P et al. (2017). Secretory IgA deficiency in individual small airways is associated with persistent inflammation and remodeling. American Journal of Respiratory and Critical Care Medicine 195: 1010-1021. DOI 10.1164/ rccm.201604-0759OC.

Pandya MC, Callahan SM, Savchenko KG, Stobart CC (2019). A contemporary view of respiratory syncytial virus (RSV) biology and strain-specific differences. Pathogens 8: 67. DOI 10.3390/pathogens8020067.

Salimi V, Ramezani A, Mirzaei H, Tahamtan A, Faghihloo E et al. (2017). Evaluation of the expression level of 12/15 lipoxygenase and the related inflammatory factors (CCL5, CCL3) in respiratory syncytial virus infection in mice model. Microbial Pathogenesis 109: 209-213. DOI 10.1016/j.micpath.2017.05.045.

Tsubaki M, Takeda T, Kino T, Itoh T, Imano $M$ et al. (2015). Mangiferin suppresses CIA by suppressing the expression of TNF- $\alpha$, IL-6, IL-1 $\beta$, and RANKL through inhibiting the activation of NF- $\kappa \mathrm{B}$ and ERK1/2. American Journal of Translational Research 7: 1371.

Wang C, Xu J, Yang L, Xu Y, Zhang X et al. (2018). Prevalence and risk factors of chronic obstructive pulmonary disease in China (the China Pulmonary Health $[\mathrm{CPH}]$ study): A national cross-sectional study. Lancet 391: 1706-1717. DOI 10.1016/S0140-6736(18)30841-9.

Wang R, Lu B, Gerard C, Gerard NP (2016). C5L2, the second C5a anaphylatoxin receptor, suppresses LPS-induced acute lung injury. American Journal of Respiratory Cell and Molecular Biology 55: 657-666. DOI 10.1165/rcmb.2016-0067OC.

Wang X, Wu K, Keeler SP, Mao D, Agapov EV et al. (2021). TLR3activated monocyte-derived dendritic cells trigger progression from acute viral infection to chronic disease in the lung. Journal of Immunology 206: 1297-1314. DOI 10.4049/jimmunol.2000965. 